\title{
AN ALGORITHM FOR THE TOPOLOGICAL DEGREE OF A MAPPING IN $n$-SPACE ${ }^{1}$
}

\author{
BY FRANK STENGER
}

Communicated by Eugene Isaacson, October 3, $1974^{2}$

1. Introduction. In this paper we announce a new formula for computing the topological degree $d(F, P, \theta)$, where $F=\left(f^{1}, \cdots, f^{n}\right)$ is a vector of real continuous functions mapping a polyhedron $P$ in $R^{n}$ into $R^{n}$, and $\theta$ is the zero vector in $R^{n}$.

Let $A=\left[a_{i j}\right]$ be an $n \times n$ real matrix, and let $A_{i}$ denote the $i$ th row of $A$. We use the convenient notation $\Delta_{n}\left(A_{1}, \cdots, A_{n}\right)$ for the determinant of $A$, and $\left|A_{i}\right| \equiv\left(a_{i 1}^{2}+\cdots+a_{i n}^{2}\right)^{1 / 2}$ for the Euclidean norm of $A_{i}$.

Let $X_{0}, X_{1}, \cdots, X_{q}$ denote $q+1$ points in $R^{n}$, where $q \leqslant n$, such that the vectors $X_{i}-X_{0}, i=1,2, \cdots, q$, are linearly independent. A $q$-simplex with vertices at $X_{0}, \cdots, X_{q}$ is defined by

$$
S_{q}\left(X_{0}, \cdots, X_{q}\right) \equiv\left\{X \in R^{n}: X=\sum_{i=0}^{q} \lambda_{i} X_{i}, \lambda_{i} \geqslant 0, \sum_{i=0}^{n} \lambda_{i}=1\right\} .
$$

We denote by $\left[X_{0} \cdots X_{q}\right]$ the oriented $q$-simplex, defined as in [2]. For example, if $q=n$, then $\left[X_{0} \cdots X_{q}\right]=\left[X_{0} \cdots X_{n}\right]$ is said to be positively (negatively) oriented in $R^{n}$ if $\Delta_{n+1}\left(Z_{0}, \cdots, Z_{n}\right)>0(<0)$, where $Z_{i}=$ $\left(1, X_{i}\right)$.

Let $\boldsymbol{P}$ be a connected, $n$-dimensional closed polyhedron represented as a "sum" of $m^{\prime}$ positively oriented $n$-simplexes in the form

$$
P=\sum_{j=1}^{m^{\prime}}\left[X_{0}^{(j)} \cdots X_{n}^{(j)}\right]
$$

such that the intersection of any two of the simplexes has zero $n$-dimensional volume.

AMS (MOS) subject classifications (1970). Primary 55C25; Secondary 55C20, 65H05, 65H10, 68A10.

Key words and phrases. Topological degree, algorithm, nonlinear equations.

Work supported by U. S. Army Research Grant \#DAHC-04-G-017.

2 Originally received July 2, 1974. 
The boundary of $\left[X_{0} \cdots X_{n}\right]$ is represented in terms of oriented $n-1$-simplexes by

$$
b\left[X_{0} \cdots X_{n}\right]=\sum_{i=0}^{n}(-1)^{i}\left[X_{0} \cdots X_{i-1} X_{i+1} \cdots X_{n}\right]
$$

(see [2]). By means of this expansion, the boundary of $P$ may be represented in the form

$$
b(P)=\sum_{j=1}^{m} t_{j}\left[Y_{1}^{(j)} \cdots Y_{n}^{(j)}\right]
$$

where $P$ is defined in (1.1), and $t_{j}= \pm 1$. For example, if $n=1$,

$$
\begin{gathered}
P=\left[X_{0} X_{1}\right]+\left[X_{1} X_{2}\right]+\cdots+\left[X_{m-1} X_{m}\right], \\
b(P)=\left[X_{m}\right]-\left[X_{0}\right] .
\end{gathered}
$$

Let $F$ be a vector of $n$ real $C^{1}$ functions defined on $P$, such that $F \neq \theta=(0, \cdots, 0)$ on $b(P)$. We denote by $d(F, P, \theta)$ the topological degree of $F$ at $\theta$ relative to $P$. We define $d(F, P, \theta)$ by

$$
d(F, P, \theta)=\frac{1}{2}\left\{\frac{F\left(X_{m}\right)}{\left|F\left(X_{m}\right)\right|}-\frac{F\left(X_{0}\right)}{\left|F\left(X_{0}\right)\right|}\right\} \quad \text { if } n=1 \text {, }
$$

$$
d(F, P, \theta)=\frac{1}{\Omega_{n-1}} \int_{b(P)} \frac{1}{|F|^{n}} \Delta_{n}\left(F, \frac{\partial F}{\partial u^{1}}, \cdots, \frac{\partial F}{\partial u^{n-1}}\right) d u^{1} \cdots d u^{n-1}
$$

if $n>1$,

where $\Omega_{n-1}$ denotes the $n-1$ dimensional volume of the surface of the $n$-sphere, and where $F=F(X(U))$ is suitably parametrized as a function of $U=\left(u^{1}, \cdots, u^{n-1}\right)$ (see [1, pp. 465-467]). If $F$ is merely real and continuous on $P$, but not necessarily of class $C^{1}$, we define $d(F, P, \theta)$ by $d(F, P, \theta)=\lim _{(\nu \rightarrow \infty)} d\left(F^{(\nu)}, P, \theta\right)$, where $F^{(\nu)}$ is real and of class $C^{1}$ on $P$ for $\nu=1,2, \cdots, \max _{(X \in P)}\left|F(X)-F^{(\nu)}(X)\right| \rightarrow 0$ as $\nu \rightarrow \infty$, and $d\left(F^{(\nu)}, P, \theta\right)$ is defined by means of (1.4).

The integral formula (1.4) is due to Kronecker [1, pp. 465-467]. Another integral for $d(F, P, \theta)$ has been given by Heinz [3]. In the following section we shall describe another procedure for evaluating $d(F, P, \theta)$, which depends only on the sign of the components of $F$ at a finite number of points of $b(P)$. 
2. Formula for $d(F, P, \theta)$. If $a$ is a real number, we define $\operatorname{sgn} a$ by $\operatorname{sgn} a=-1,0$ or 1 if $a<0,=0$ or $>0$ respectively. We define $\operatorname{sgn} F$ by $\operatorname{sgn} F=\left(\operatorname{sgn} f^{1}, \cdots, \operatorname{sgn} f^{n}\right)$. Let us set

$$
\delta_{m}(F, P, \theta)=\frac{1}{2^{n} n !} \sum_{j=1}^{m} t_{j} \Delta_{n}\left(\operatorname{sgn} F\left(Y_{1}^{(j)}\right), \cdots, \operatorname{sgn} F\left(Y_{n}^{(j)}\right)\right)
$$

where the $t_{j}$ and $Y_{i}^{(j)}$ are the same as in (1.2). This formula is used to compute $d(F, P, \theta)$ by means of the following

Algorithm 2.1. (1) Let $p$ be a fixed positive integer.

(2) Set $\delta=\delta_{m}(F, P, \theta)$ as defined in (2.1).

(3) Revise the definition of $b(P)$ as follows: For $j=1,2, \cdots, m$,

(a) locate the longest segment $\overline{Y_{k}^{(j)} Y_{l}^{(j)}}(k<l)$ of the oriented simplex $t_{j}\left[Y^{(j)} \cdots Y_{n}^{(j)}\right]$ in (1.2), and set $A=\left(Y_{k}^{(j)}+Y_{l}^{(j)}\right) / 2$;

(b) replace $t_{j}\left[Y_{1}^{(j)} \cdots Y_{n}^{(j)}\right]$ according to:

$$
\begin{aligned}
t_{j}\left[Y_{1}^{(j)} \cdots Y_{k}^{(j)} \cdots Y_{l}^{(j)} \cdots Y_{n}^{(j)}\right] & \leftarrow t_{j}\left[Y_{1}^{(j)} \cdots A \cdots Y_{l}^{(j)} \cdots Y_{n}^{(j)}\right] \\
t_{j+m}\left[Y_{1}^{(j+m)} \cdots Y_{k}^{(j+m)} \cdots Y_{l}^{(j+m)} \cdots Y_{n}^{(j+m)}\right] & \\
& \leftarrow t_{j}\left[Y_{1}^{(j)} \cdots Y_{k}^{(j)} \cdots A \cdots Y_{n}^{(j)}\right]
\end{aligned}
$$

(4) replace $m$ by $2 m$ to get a new decomposition of $b(P)$ in terms of (twice as many) oriented simplexes.

(5) Set $e=\delta_{m}(F, P, \theta)$ as defined in (2.1), with the new $b(P)$.

(6) If $\delta=e=$ integer, go to Step 6. Otherwise set $\delta=e$ and return to Step 3.

(7) Replace $p$ by $p-1$. If the resulting $p$ is positive, return to Step 3. Otherwise print out $m, \delta$.

Let us now make the following

Assumption 2.2. Let $F$ be continuous and real on $P$, where $P$ is defined as in equation (1.1). Let $b(P)$ be defined as in equation (1.2), and let $F \neq \theta$ on $b(P)$. If $n>1$, for all $1<\mu \leqslant n, \varphi^{i}=f^{j_{i}}, j_{k} \neq j_{l}$ if $k \neq l$, and $\Phi_{\mu}=\left(\varphi^{1}, \cdots, \varphi^{\mu}\right)$, we assume that the sets $T\left(A_{\mu}\right)=\{X \in b(P)$ : $\left.\Phi_{\mu}(X) /\left|\Phi_{\mu}(X)\right|=a_{\mu}\right\} \cap S_{\mu-1}$ and $b(P)-T\left(A_{\mu}\right)$ consist of a finite number of connected subsets of $b(P)$, for all vectors $a_{\mu}=( \pm 1,0, \cdots, 0),(0, \pm 1,0, \cdots, 0), \cdots$, $(0, \cdots, 0, \pm 1)$, and for all $\mu-1$-simplexes $S_{\mu-1}$ on $b(P)$.

THEOREM 2.3. If Assumption 2.2 is satisfied and if the integer $p$ in Algorithm 2.2 is chosen sufficiently large, then Algorithm 2.1 prints out 
finite integers $m$ and $\delta$, where $\delta=d(F, P, \theta)$, and where $P$ is defined in (1.1).

\section{REFERENCES}

1. P. Alexandroff and H. Hopf, Topologie. I, Springer-Verlag, New York, 1935.

2. J. Cronin, Fixed points and topological degree in nonlinear analysis, Math. Surveys, no. 11, Amer. Math. Soc., Providence, R. I., 1964. MR 29 \#1400.

3. E. Heinz, An elementary analytic theory of the degree of a mapping in n-dimensional space, J. Math. Mech. 8 (1959), 231-247. MR 21 \#1370.

DEPARTMENT OF MATHEMATICS, UNIVERSITY OF UTAH, SALT LAKE CITY, UTAH 84112 